\title{
Scolia
}

Revue de linguistique

Espace et temps : quelles asymétries ?

\section{John Humbley, La néologie terminologique, Limoges}

\section{Teresa Lino}

\section{OpenEdition \\ Journals}

Édition électronique

URL : http://journals.openedition.org/scolia/995

DOI : $10.4000 /$ scolia.995

ISSN : 2677-4224

\section{Éditeur}

Presses universitaires de Strasbourg

\section{Édition imprimée}

Date de publication : 11 juillet 2019

Pagination : 167-169

ISBN : 979-1-03440-050-8

ISSN : $1253-9708$

\section{Référence électronique}

Teresa Lino, «John Humbley, La néologie terminologique, Limoges », Scolia [En ligne], 33 | 2019, mis en ligne le 11 juillet 2019, consulté le 25 septembre 2020. URL : http://journals.openedition.org/scolia/ 995 ; DOl : https://doi.org/10.4000/scolia.995 
John HUMBLEY, La néologie terminologique, Limoges, Éditions LambertLucas, collection La Lexicothèque, 2018, 468 pages.

John Humbley est professeur émérite à la Sorbonne Paris Université, membre du CILLAC-ARP (EA 3967), cofondateur de la revue Neologica, et professeur invité à l'Université de Vérone. Il est reconnu en France et dans beaucoup d'institutions universitaires pour ses recherches sur la terminologie et la néologie, depuis une trentaine d'années.

Selon l'auteur, cet ouvrage sur «la néologie terminologique» essaie de combler une lacune: "L'importance de la néologie dans le cadre de la recherche en terminologie n'a jamais fait de doute: elle est reconnue dans les manuels de terminologie, surtout les plus anciens. En plus, pour le public francophone de part et d'autre de l'Atlantique, terminologie et néologie sont devenues des quasi-synonymes. Il était donc étonnant de constater qu'aucune monographie ne semblait avoir été consacrée au sujet. L'ambition de ce livre est de combler - tant que faire se peut - cette lacune» (p. 13).

Le livre est organisé en deux parties: la première d'ordre encyclopédique a pour objectif une réflexion sur la néologie et ses enjeux dans les langues de spécialité; l'auteur fait une présentation exhaustive des études, des principales théories de nombreux auteurs dans des contextes scientifiques, pratiques et institutionnels, en France, au Canada et dans d'autres pays; les citations abondantes soulignent les différents points de vue qui cherchent à rendre compte du processus néologique tel qu'il se produit dans le domaine terminologique. La seconde partie a pour objet, d'une part, l'observation de trois vocabulaires en voie de constitution - l'un du XIX ${ }^{\mathrm{e}}$ siècle, les deux autres de la fin du $x^{\mathrm{e}}$ siècle - et, d'autre part, la mise à l'épreuve de certains principes de néologie.

L'auteur s'interroge sur «comment parler de néologie en terminologie?» (cf. chap. $1 \$ 1.6$ p. 35-36). Ce chapitre évoque (cf. p. 17) la problématique de l'apparition de nouveaux termes, préoccupation des scientifiques du XVIII ${ }^{\mathrm{e}}$ siècle, en sciences naturelles et en chimie qui est devenue une thématique fondamentale en terminologie théorique et pratique au $\mathrm{xx}^{\mathrm{e}}$ et $\mathrm{xxI}^{\mathrm{e}}$ siècles. Une synthèse rigoureuse présente les nombreuses études et les différentes approches sur la néologie ainsi que les tentatives de leur théorisation surtout francophone des années 
1960, 1970 et 1980; mais c'est au début du Xxi ${ }^{\mathrm{e}}$ siècle que l'étude de la néologie repart sur de nouvelles bases théoriques et méthodologiques.

Les «Prémisses néologiques et terminologiques» présentées au chapitre 2 (cf. $\$ 2.1$ p. 37 ) soulignent que la néologie terminologique peut avoir au moins deux approches: la néologie terminologique en tant que «catégorie» de la néologie ou "relevant de la terminologie». La nature de ces deux phénomènes conduit l'auteur à passer en revue les principales théories de la néologie et la terminologie ainsi que les concepts de néologisme et de terme. D’autres notions sont passées en revue telles que celles de concept, de néonymie et de néonyme, motivation, synonymie en terminologie, de définition (en terminologie), diachronie et synchronie (en néologie).

Avant de présenter quelques hypothèses sur la description de la néologie terminologique, l'auteur adopte une vision scalaire à la fois de la néologie et de la terminologie étant donné la variété des approches: «un élément peut ainsi être plus ou moins néologique, plus ou moins terminologique» (p. 81).

Dans la partie du chapitre 4 dédiée «à la recherche d'un modèle de néologie terminologique» (p. 101), l'auteur ébauche quatre modèles de néologie terminologique qui répondent à la question: "comment rendre compte de l'apparition de nouveaux termes?». Le premier modèle appelé provisoirement «modèle incrémental» s'inspire des travaux de Kageura (2002) qui "postule que les termes nouveaux se construisent à partir des termes existants d'un domaine». Le deuxième, le «modèle de discursif» s'inspire de plusieurs courants de la linguistique fonctionnelle systémique et, plus particulièrement, des travaux de Halliday (2004); selon cette hypothèse, les néologismes terminologiques se forment en discours et finissent par être lexicalisés et retenus par les spécialistes puisqu'ils «encapsulent» les nouveaux termes. Le troisième, le "modèle métaphorique» a pour base quelques principes de la linguistique cognitive, défendus par certains auteurs, dont Temmermann (2000); cependant cette auteure est plus ambitieuse puisqu'elle cherche à expliquer les mécanismes des processus cognitifs sous-jacents aux découvertes scientifiques. Le quatrième, un «modèle mixte » en construction, s'applique à un contexte diachronique.

Dans la seconde partie de l'ouvrage, aux chapitres 8 et 12 sont examinés trois domaines néologiques: deux domaines récents (la nouvelle économie, le commerce électronique) et un troisième, celui 
de la reproduction sonore, qui illustre «la dimension diachronique ou rétrospective» (p. 202).

Les différents types de normalisation terminologique (officielle, scientifique ou professionnelle) ainsi que la politique de néologie terminologique officielle des pays francophones (France et Québec) font l'objet du chapitre 9.

Les thématiques de politique linguistique, de néologie terminologique et de développement terminologique sont présentées dans le chapitre 10, où l'auteur présente de nombreuses informations sur plusieurs langues de différents pays d'Asie et d'Afrique.

Le chapitre 11 est dédié à plusieurs sujets dont la néologie de transfert, l'emprunt et la néologie intralinguistique.

Cet ouvrage de référence se termine par une réflexion très importante pour tous ceux qui travaillent en néologie terminologique: «Il semble donc raisonnable de ne pas exclure la création consciente de la néologie terminologique [...] l'inconscience dans ce cas ne concerne que la fonction de nomination: on peut supposer un choix conscient de l'effet de communication immédiate visée, la raison même de la néologie de type discursif. Mais il est clair que la néologie terminologique n'est que relativement plus consciente que celle de la langue générale, compte tenu de ses besoins de nomination» (p. 399).

Teresa LINO

Universidade Nova de Lisboa,

Groupe de Lexicologie, Lexicographie et de Terminologie unl.tlino@mail.telepac.pt

\section{Références}

HALLIDAY M.A.K. (2004), Langage and Knowledge: the 'Unpacking' of Text, in Webster J. (ed.), The Langage of Science, London/ New York, Continuum, (first edition 1998), 49-101.

KAGEURA K. (2002), The Dynamics of Terminology. A Descriptive Theory of Term and Formation and Terminological Growth, Amsterdam and Philadelphia, Benjamins.

TEMMERMANN R. (2000), Towards New Ways of Terminology Description. The Sociocognitive Approach, Amsterdam and Philadelphia, Benjamins. 